\title{
Commentaire
}

\section{Propagation de protéines mal repliées : Nouvelles possibilités thérapeutiques, nouveau risque pour la santé publique}

\author{
Cashman, NR ${ }^{1 *}$ \\ ${ }^{1}$ Faculté de médecine, Université de la Colombie-Britannique, Vancouver (Colombie-Britannique) \\ *Correspondance : neil.cashman@vch.ca
}

\section{Résumé}

Il existe aujourd'hui un large consensus selon lequel la propagation de protéines mal repliées est le mécanisme sous-jacent des maladies infectieuses à prions (maladie de Creutzfeldt-Jakob chez l'humain, tremblante du mouton chez la chèvre et le mouton, encéphalopathie spongiforme bovine chez les bovins et maladie débilitante chronique chez le cerf et le wapiti). Au cours des dix dernières années, il est devenu de plus en plus évident que d'autres maladies, y compris la maladie d'Alzheimer, la maladie de Parkinson et la sclérose latérale amyotrophique, peuvent évoluer selon le même mécanisme, à partir d'un polypeptide propre à chacune plutôt que d'une protéine à prion. Les publications récentes sur ces maladies neurodégénératives qui ne sont pas à prions soulignent en outre l'existence de multiples « souches » qui s'expriment différemment dans différents contextes en donnant lieu à une diversité de phénotypes. La cause probable de ces maladies neurodégénératives est maintenant désignée collectivement comme étant la « propagation de protéines mal repliées. » Ce phénomène offre de nombreuses possibilités en matière de nouveaux diagnostics et traitements. Il présente par contre un risque théorique de transmission iatrogène, bien que les études expérimentales corroborant cette notion soient limitées à l'heure actuelle.

\section{Introduction}

L'hypothèse qu'une protéine mal repliée pourrait transférer son anomalie à une protéine voisine qui est normale et provoquer une maladie a été largement considérée comme une hérésie lorsque Stanley Prusiner (1) l'a proposée pour la première fois en 1982, mais son idée est maintenant bien acceptée et lui a valu le prix Nobel de médecine 1997. II existe aujourd'hui un large consensus selon lequel la propagation sous forme mal repliée d'une protéine de l'hôte (codée chez l'humain par le gène PRNP) est le mécanisme sous-jacent des maladies infectieuses à prions (maladie de Creutzfeldt-Jakob chez l'humain, tremblante du mouton chez la chèvre et le mouton, encéphalopathie spongiforme bovine chez les bovins et maladie débilitante chronique chez le cerf et le wapiti). Plus récemment, il est devenu évident que le mauvais repliement des protéines pourrait faire partie de la pathologie sous-jacente dans les maladies neurodégénératives plus courantes telles que la maladie d'Alzheimer, la maladie de Parkinson et la sclérose latérale amyotrophique, et possiblement dans certaines affections non neurologiques telles que le diabète sucré de type II et l'améloïdose périphérique (2).

Le présent article a pour objectif de présenter quelques faits saillants de la façon dont les polypeptides influencent le mauvais repliement des protéines qui ne sont pas de type prion dans les maladies neurodégénératives. II vise à explorer les possibilités de diagnostic et de traitement et à déterminer le risque théorique de transmission iatrogène qui serait impliqué. L'encadré ci-dessous présente une terminologie courante des protéines mal repliées. 


\section{Terminologie des protéines mal repliées}

Dissémination de la PPMR. ${ }^{1}$ Application de la théorie d'agrégation des prions à la propagation d'agrégats de protéines mal repliées; nécessite un événement spécifique d'agrégation de monomères mal repliés dans un modèle de production (cinétique lente) pour le recrutement de monomères supplémentaires (cinétique rapide).

Épitope. Pour les protéines, région ou séquence d'acides aminés pouvant être reconnue par un anticorps spécifique.

Exosomes. Petites ( $100 \mathrm{~nm})$ vésicules membranaires sécrétées par les cellules vivantes; contiennent des protéines et des acides nucléiques.

Modification post-traductionnelle par liaison covalente. Tout changement survenant dans une protéine médiée par une liaison chimique, comme l'oxydation et la glycosylation.

Prions. Agrégats de protéines infectieuses, responsables de la transmission de la maladie de Creutzfeldt-Jakob, de la tremblante du mouton, de l'encéphalopathie spongiforme bovine et de la maladie débilitante chronique des cervidés.

Propagation de protéines mal repliées. Transmission de protéines mal repliées de type prion entre les cellules dans un organe comme le cerveau, médiée par des agrégats de protéines et les exosomes. Bien que de type prion, aucune donnée à ce jour ne démontre la transmission naturelle de maladie chez une espèce ou d'une espèce à l'autre.

Souches de PPMR. ${ }^{1}$ Application d'un comportement de souche prion à la propagation de protéines mal repliées qui ne sont pas de type prion, en corrélation avec les différentes structures des agrégats de protéines touchés par la propagation.

PPMR = propagation de protéines mal repliées

\section{Mauvais repliement de prions et d'autres polypeptides}

Nous savons maintenant que des protéines mal repliées peuvent se propager, soit par l'intermédiaire des prions infectieux constitués d'agrégats mal repliés de la protéine prion de l'hôte, soit par la propagation d'agrégats d'autres protéines impliquées dans les maladies neurodégénératives (aucun cycle évolutif naturel de l'infection). De récents essais ont démontré, par exemple, que la propagation de certaines protéines mal repliées est maintenant liée aux troubles neurodégénératifs suivants : oligomères ou fibrilles bêta-amiloïdes ( $\mathrm{A}$ bêta) pour la maladie d'Alzheimer (3); protéine alpha-synucléine pour la maladie de Parkinson, la démence à corps de Lewy (4) et l'atrophie multisystématisée (5); superoxyde dismutase 1 (SOD1) (6) et protéine transactivatrice (TAR) de liaison à l'ADN 43 (TDP-43) (7) pour la sclérose latérale amyotrophique; protéine tau pour les tauopathies, ainsi que la maladie d'Alzheimer (8). Les maladies neurodégénératives, impliquant ou non une infection à prion, progressent avec la propagation de protéines mal repliées.

\section{Différentes souches de propagation de protéines mal repliées influencent différentes} progressions des maladies

Les prions infectieux (maladie de Creutzfeldt-Jakob, tremblante du mouton, encéphalopathie spongiforme bovine et maladie débilitante chronique) n'existent pas sous forme monolithique, mais peuvent adopter un comportement de « souche ». Dans le cas de la tremblante du mouton, par exemple, lors de la première transmission de prions infectieux à des modèles murins, environ 20 souches ont été clonées. Elles ont été définies en fonction de la période d'incubation, des régions du cerveau principalement touchées et de caractéristiques biochimiques comme le schéma de préférences de glycosylation. Tout récemment, il a été démontré que, à l'instar de la propagation de protéines mal repliées, les agents non infectieux de type prion qui causent des maladies neurodégénératives présentent aussi des comportements de souche. Des études portant 
sur la maladie d'Alzheimer ont établi une corrélation entre les propriétés de souche d'agrégats A bêta dans le cerveau humain et les taux de progression de la maladie $(9,10)$. Pour la maladie de Parkinson et l'atrophie multisystématisée, différentes souches d'agrégats de la protéine alpha synucléine ont été observées in vitro et in vivo (11). Dans le cas de la sclérose latérale amyotrophique, il a été démontré que la propagation de SOD1 présentait au moins deux formes distinctes chez un modèle murin (12). Et la tauopathie a révélé une propagation à souches multiples (13) qui correspondent aux caractéristiques et aux taux de progression de la maladie selon les observations cliniques.

Nouveaux diagnostics et traitements pour les maladies liées aux protéines mal repliées Une nouvelle mentalité s'impose envers le développement de médicaments pour les maladies liées aux protéines mal repliées. Pendant plus d'un siècle, la science pharmacologique a très bien réussi à appliquer de petites molécules à des structures fixes : il s'agit d'une stratégie efficace pour le ciblage sélectif des canaux, des pores ainsi que des poches et échancrures à la surface des enzymes qui peuvent induire une modification de l'activité protéique. En général cependant, l'approche fondée sur les petites molécules ne s'applique pas aux protéines mal repliées en raison de l'ampleur et de l'instabilité fréquentes des cibles. De plus, les maladies liées à la propagation de protéines mal repliées impliquent des interactions protéine-protéine qui s'avèrent très difficiles à cibler avec des médicaments.

L'identification des souches ouvre la porte au traitement des maladies neurodégénératives à l'aide de la « médecine de précision ». Mais il y a un inconvénient : les données accumulées indiquent que le traitement d'une souche de prions infectieux peut devenir « sélectif » pour une autre souche au pouvoir pathogène plus grand. Ce précédent des maladies infectieuses à prions doit nous sensibiliser à la possibilité que le blocage de la propagation de protéines mal repliées dans d'autres maladies neurodégénératives soit aussi sélectif de souches émergentes qui nécessiteront leur propre traitement.

Nos travaux prévoient que le diagnostic et le traitement efficaces des maladies liées aux protéines mal repliées imposent un nouveau paradigme fondé sur la détermination rationnelle d'épitopes sélectifs (ou cibles des anticorps) des protéines mal repliées qui ont un rôle déterminant dans le processus pathogénique. Plusieurs mécanismes rendent efficace le ciblage sélectif des protéines mal repliées par des anticorps, y compris la neutralisation de la cytotoxicité des agrégats et l'inhibition de la propagation de protéines mal repliées de type prion (14).

Dans certaines conditions, comme une maladie à prion infectieuse et la maladie d'Alzheimer, le ciblage précis de la propagation de protéines mal repliées ressemble à « trouver une aiguille dans une botte de foin » alors que des milliers sinon des millions d'espèces mal repliées peuvent être présentes en plus faible concentration que l'espèce repliée originellement, une situation que nous avons qualifiée de « distraction de la cible ». Toutefois, l'avantage de l'immunothérapie spécifique pour les protéines mal repliées c'est qu'ainsi les isoformes de protéines repliées normalement n'auront pas à subir une reconnaissance immunologique du soi.

Une perte ou un gain de structure peut produire un mauvais repliement d'épitopes spécifiques Deux mécanismes des maladies liées aux protéines mal repliées peuvent provoquer un mauvais repliement d'épitopes immunologiques ou biomarqueurs de maladies, soit : une perte ou un gain de structure des isoformes des protéines normales. Un gain de structure peut survenir lors de la génération de néoépitopes découlant d'une modification post-traductionnelle par liaison covalente (médiée par la formation d'une liaison chimique). Des forces non covalentes peuvent engendrer des néoépitopes également. Nous avons, par exemple, pu identifier de nouveaux épitopes provenant d'un gain de structure qui ont été générés par des forces non covalentes sous-jacentes dans des agrégats de morphologies spécifiques, comme les oligomères $A$ bêta dans la maladie d'Alzheimer (15).

II peut y avoir perte de structure lorsqu'un domaine structuré d'une protéine « se relâche » en révélant une séquence linéaire qui peut être reconnue par un anticorps généré contre la séquence peptidique libre. Nous avons élaboré des méthodes rationnelles pour prédire les épitopes propres à une maladie en raison d'une perte de structure, dont les résultats sont des séquences de peptides linéaires passant de structurées à non structurées dans la protéine mal repliée et pour lesquels il est possible de générer et de sélectionner des 
anticorps en fonction du peptide cible et dans le contexte de la protéine mal repliée de la maladie. Par exemple, la génération d'hypothèses et des approches computationnelles ont permis de repérer des épitopes spécifiques mal repliés dans la SOD1 afin de bloquer la toxicité et la propagation de la SOD1 mal repliée de type prion dans la sclérose latérale amyotrophique $(16,17)$.

\section{Risque théorique de transmission iatrogène}

II ne fait aucun doute que de véritables prions infectieux peuvent être détectés dans le sang et sont capables de transmettre la maladie (18). En effet, dans le cas de variantes de la maladie de Creutzfeldt-Jakob, cinq transmissions de la maladie par du sang ou des produits sanguins sont bien documentées (19). En revanche, la présence de sources de dissémination de protéines mal repliées dans les maladies neurodégénératives qui ne sont pas à prions apparaît moins clairement. Un journal seulement a publié un article sur les oligomères $A$ bêta dans la maladie d'Alzheimer (20).

Quelques rapports scientifiques (y compris des présentations scientifiques au cours de réunions et des demandes de brevets) laissent entendre que la pathologie de la maladie d'Alzheimer peut être transmise à diffusion hématogène chez les animaux de laboratoire. Des oligomères de la protéine alpha synucléine ont été détectés dans le plasma de patients atteints de la maladie de Parkinson, mais ont été aussi observés à des niveaux similaires lors de contrôles normaux, ce qui donne à penser que l'analyte détecté n'est pas pathogène (21). II n'existe aucun rapport traitant des oligomères SOD1 ou TDP-43 dans le sang périphérique ou du sérum, peut-être en raison d'une sensibilité inadéquate ou d'une dissémination dans le sang par d'autres voies, comme les exosomes (17). Fait intéressant, un seul rapport montre, chez les personnes ayant reçu des injections d'hormones de croissance provenant de dons cadavériques, une incidence plus élevée de sclérose latérale amyotrophique, mais pas de la maladie d'Alzheimer ou de la maladie de Parkinson (22). Ainsi, il est possible qu'un risque théorique réside dans la transmission iatrogène de maladies neurodégénératives, mais il existe peu d'études expérimentales et épidémiologiques à l'appui.

\section{Conclusions}

Une nouvelle ère dans le diagnostic et le traitement des maladies liées à la propagation de protéines mal repliées s'ouvre devant nous. Elle offre de nombreuses possibilités de traiter des maladies neurodégénératives auparavant incurables ou mal traitées. Certaines immunothérapies destinées à bloquer la propagation et la toxicité de protéines mal repliées renferment un potentiel thérapeutique. Le spectre de la transmission iatrogène de la sclérose latérale amyotrophique, de la maladie d'Alzheimer, de la maladie de Parkinson et d'autres syndromes neurodégénératifs représente un risque théorique. À l'heue actuelle, il existe peu ou pas de soutien pour des sources de dissémination de maladies non à prions liées à la propagation de protéines mal repliées qui seraient détectables dans le sang périphérique ou de paradigmes expérimentaux à l'appui de cette perspective inquiétante.

\section{Conflit d'intérêts}

$D^{r}$ Neil Cashman préside la Chaire de recherche du Canada en neurodégénérescence et en maladies liées aux protéines mal repliées. Il est le fondateur et le chef des affaires scientifiques de ProMIS Neurosciences Inc.

\section{Références}

(1) Prusiner SB. Novel proteinaceous infectious particles cause scrapie. Science. 1982;216(4542):136-44.

(2) Moreno-Gonzalez I, Soto C. Misfolded protein aggregates: Mechanisms, structures and potential for disease transmission. Semin Cell Dev Biol. 2011 Jul;22(5):482-7.

(3) Kane MD, et al. Evidence for seeding of beta-amyloid by intracerebral infusion of Alzheimer brain extracts in betaamyloid precursor protein-transgenic mice. J Neurosci. 2000;20(10):3606-11.

(4) Luk KC, et al. Intracerebral inoculation of pathological alpha-synuclein initiates a rapidly progressive neurodegenerative alpha-synucleinopathy in mice. J Exp Med. 2012;209(5):975-86. 
(5) Watts JC, Giles K, Oehler A, et al. Transmission of multiple system atrophy prions to transgenic mice. Proc Natl Acad Sci USA. 2013;110(48):19555-60.

(6) Grad LI, et al. From molecule to molecule and cell to cell: Prion-like mechanisms in amyotrophic lateral sclerosis. Neurobiol Dis. 2015 May;77:257-65.

(7) Brettschneider J, Arai K, Del Tredici K, et al. TDP-43 pathology and neuronal loss in amyotrophic lateral sclerosis spinal cord. Acta Neuropathol. 2014;128(3):423-37.

(8) Frost B, Diamond MI. Prion-like mechanisms in neurodegenerative diseases. Nat Rev Neurosci. 2010;11(3):155-9.

(9) Watts JC, Condello C, Stohr J, et al. Serial propagation of distinct strains of Abeta prions from Alzheimer's disease patients. Proc Natl Acad Sci USA. 2014;111(28):10323-8.

(10) Stohr J, Condello C, Watts JC, et al. Distinct synthetic Abeta prion strains producing different amyloid deposits in bigenic mice. Proc Natl Acad Sci USA. 2014;111(28):10329-34.

(11) Guo JL, Covell DJ, Daniels JP, et al. Distinct alpha-synuclein strains differentially promote tau inclusions in neurons. Cell. 2013;154(1):103-17.

(12) Ayers JI, Fromholt S, Koch M, et al. Experimental transmissibility of mutant SOD1 motor neuron disease. Acta Neuropathol. 2014;128(6):791-803.

(13) Sanders DW, Kaufman SK, DeVos SL, et al. Distinct tau prion strains propagate in cells and mice and define different tauopathies. Neuron. 2014;82(6):1271-88.

(14) Guest WC, Silverman JM, Pokrishevsky E, et al. Generalization of the prion hypothesis to other neurodegenerative diseases: An imperfect fit. J Toxicol Environ Health A. 2011;74(22-24):1433-59.

(15) Silverman J, Gibbs E, Rezaei H, et al. An immunological epitope specific for toxic oligomeric Abeta in Alzheimer's disease. Alzheimer's \& Dementia: The Journal of the Alzheimer's Association. 2012;8(4):P625.

(16) Grad LI, Yerbury JJ, Turner BJ, et al. Intercellular propagated misfolding of wild-type Cu/Zn superoxide dismutase occurs via exosome-dependent and -independent mechanisms. Proc Natl Acad Sci USA. 2014;111(9):3620-5.

(17) Grad LI, Guest WC, Yanai A, et al. Intermolecular transmission of superoxide dismutase 1 misfolding in living cells. Proc Natl Acad Sci USA. 2011;108(39):16398-403.

(18) Wood H. Prion disease: New techniques developed for prion detection in blood and cerebrospinal fluid. Nat Rev Neurol. 2011;7(4):183.

(19) Lefrere JJ, Hewitt P. From mad cows to sensible blood transfusion: The risk of prion transmission by labile blood components in the United Kingdom and in France. Transfusion. 2009;49(4):797-812.

(20) Zhang J, Peng M, Jia J. Plasma amyloid-beta oligomers and soluble tumor necrosis factor receptors as potential biomarkers of AD. Curr Alzheimer Res. 2014 May;11(4):325-31.

(21) Park MJ, Cheon SM, Bae HR, et al. Elevated levels of alpha-synuclein oligomer in the cerebrospinal fluid of drugnaive patients with Parkinson's disease. J Clin Neurol. 2011 Dec;7(4):215-22.

(22) Irwin DJ, et al. Evaluation of potential infectivity of Alzheimer and Parkinson disease proteins in recipients of cadaverderived human growth hormone. JAMA Neurol. 2013 Apr;70(4):462-8. 